\title{
Philosophiques
}

\section{Fait et valeur dans le concept de trouble mental : le trouble en tant que dysfonction préjudiciable}

\section{Jerome Wakefield}

Volume 33, numéro 1, printemps 2006

Philosophie et psychopathologie

URI : https://id.erudit.org/iderudit/012946ar

DOI : https://doi.org/10.7202/012946ar

Aller au sommaire du numéro

\section{Éditeur(s)}

Société de philosophie du Québec

ISSN

0316-2923 (imprimé)

1492-1391 (numérique)

Découvrir la revue

Citer cet article

Wakefield, J. (2006). Fait et valeur dans le concept de trouble mental : le trouble en tant que dysfonction préjudiciable. Philosophiques, 33(1), 37-63.

https://doi.org/10.7202/012946ar
Résumé de l'article

Les critiques actuelles des diagnostics psychiatriques, qu'elles viennent des antipsychiatres, des béhavioristes, des constructionnistes sociaux, des szasziens et des foucaldiens, rejettent généralement l'idée que le concept de trouble mental est légitime du point de vue médical, ne laissant donc aucun argument solide à partir duquel il soit possible de mener une critique constructive et d'établir un dialogue avec la psychiatrie. Ces positions ne réussissent également pas à expliquer les fortes intuitions populaires qui permettent aux gens de distinguer les troubles psychologiques des autres conditions psychologiques négatives. Selon l'analyse de la dysfonction préjudiciable (DP) présentée dans cet article, un trouble mental est une condition : 1) préjudiciable, un concept évaluatif basé sur des valeurs sociales ; et 2) causée par une dysfonction psychologique, un concept factuel se rapportant à un mécanisme psychologique qui accomplit une fonction pour laquelle il a été biologiquement conçu (c'est-à-dire établie par la sélection naturelle). L'analyse DP est ainsi une position hybride qui requiert à la fois que des critères évaluatifs et factuels soient satisfaits pour garantir l'attribution d'un trouble. Nous argumentons que l'analyse DP réussit admirablement à expliquer les jugements intuitifs quant à ce qui est un trouble et à ce qui en n'est pas. La composante factuelle permet également de critiquer le critère diagnostique du DSM, ce que nous ferons à l'aide de plusieurs exemples (dépression, troubles du comportement). Ces critiques déterminent où le DSM a dévié de ses propres hypothèses et ainsi considéré comme des troubles ce qui n'en est pas. Elles expliquent également pourquoi les tentatives du DSM de remédier au problème en introduisant le critère du « cliniquement significatif » échouent.
Tous droits réservés @ Société de philosophie du Québec, 2006

Ce document est protégé par la loi sur le droit d'auteur. L’utilisation des services d'Érudit (y compris la reproduction) est assujettie à sa politique d'utilisation que vous pouvez consulter en ligne.

https://apropos.erudit.org/fr/usagers/politique-dutilisation/ 


\title{
Fait et valeur dans le concept de trouble mental : le trouble en tant que dysfonction préjudiciable
}

\author{
JEROME WAKEFIELD \\ New York University \\ Jerome.wakefield@nyu.edu
}

RÉSUMÉ. - Les critiques actuelles des diagnostics psychiatriques, qu'elles viennent des antipsychiatres, des béhavioristes, des constructionnistes sociaux, des szasziens et des foucaldiens, rejettent généralement l'idée que le concept de trouble mental est légitime du point de vue médical, ne laissant donc aucun argument solide à partir duquel il soit possible de mener une critique constructive et d'établir un dialogue avec la psychiatrie. Ces positions ne réussissent également pas à expliquer les fortes intuitions populaires qui permettent aux gens de distinguer les troubles psychologiques des autres conditions psychologiques négatives. Selon l'analyse de la dysfonction préjudiciable (DP) présentée dans cet article, un trouble mental est une condition : 1) préjudiciable, un concept évaluatif basé sur des valeurs sociales ; et 2) causée par une dysfonction psychologique, un concept factuel se rapportant à un mécanisme psychologique qui accomplit une fonction pour laquelle il a été biologiquement conçu (c'est-à-dire établie par la sélection naturelle). L'analyse DP est ainsi une position hybride qui requiert à la fois que des critères évaluatifs et factuels soient satisfaits pour garantir l'attribution d'un trouble. Nous argumentons que l'analyse DP réussit admirablement à expliquer les jugements intuitifs quant à ce qui est un trouble et à ce qui en n'est pas. La composante factuelle permet également de critiquer le critère diagnostique du DSM, ce que nous ferons à l'aide de plusieurs exemples (dépression, troubles du comportement). Ces critiques déterminent où le DSM a dévié de ses propres hypothèses et ainsi considéré comme des troubles ce qui n'en est pas. Elles expliquent également pourquoi les tentatives du DSM de remédier au problème en introduisant le critère $\mathrm{du}$ « cliniquement significatif » échouent.

ABSTRACT. - Current antipsychiatric, behaviorist, social-constructivist, Szaszian, and Foucauldian critiques of psychiatric diagnosis generally reject mental disorder as a medically legitimate concept, thus allowing no place to stand from which to mount a constructive critique and dialogue with psychiatry. These views also fail to explain the robust folk intuitions by which people distinguish psychological disorders from other negative psychological conditions. According to the harmful dysfunction (HD) analysis presented in this paper, a mental disorder is condition that is (1) harmful, a value concept judged by social values ; and (2) caused by a psychological dysfunction, a factual concept referring to failure of a psychological mechanism to perform a function for which it is biologically designed (i.e., naturally selected). The HD analysis is thus a hybrid account that requires both value and factual criteria to be satisfied before attribution of disorder is warranted. It is argued that the HD analysis has considerable success in explaining intuitive judgments about disorder versus non-disorder. The factual component also allows for a 
critique of DSM diagnostic criteria for specific categories, with several examples (e.g., depression, conduct disorder) discussed. These critiques identify where the DSM has deviated from its own deepest assumptions and labeled non-disorders as disorders, and why the DSM's own attempts to fix the problem, as with the «clinical significance» criterion, do not succeed.

Le concept de trouble mental est le fondement des professions de la santé mentale, et il est au cœur des querelles publiques et savantes sur les conditions considérées comme des psychopathologies. Toutefois, malgré une abondante documentation consacrée au concept de trouble mental en philosophie, en psychologie, en psychiatrie, en médecine, en sociologie et autres disciplines savantes, et malgré une abondante discussion dans la presse populaire, il n'existe toujours pas d'analyse généralement admise qui rende compte de manière adéquate des opinions largement partagées sur le trouble mental.

Une telle analyse est requise en tant que solution de rechange aux optiques stériles proposées en ce moment. D'une part, un groupe improbable d'antipsychiatres, de behavioristes, de postmodernistes et de constructivistes sociaux affirment qu'il n'y a pas de chose telle qu'un trouble mental au sens littéral de "trouble » appliqué en médecine. Ils affirment plutôt que les diagnostics de trouble mental sont simplement des jugements de valeur portant sur des catégories socialement construites de comportements désapprouvés, autorisant l'application du pouvoir médical pour un contrôle social de conditions qui ne sont pas réellement des troubles. D'autre part, l'institution psychiatrique, telle que représentée par la quatrième édition du Diagnostic and Statistical Manual of Mental Disorders (DSM, 1994) de l'Association américaine de psychiatrie, tend à concevoir que le trouble est définissable par n'importe quel syndrome comportemental négatif pouvant se manifester de manière appropriée. Nous avons désespérément besoin d'un juste milieu raisonné qui reconnaisse le fait que certaines conditions mentales sont bel et bien des troubles mentaux authentiques et qui nous explique pourquoi il en est ainsi, tout en les distinguant du vaste domaine des " problèmes existentiels ", certes déviants et socialement indésirables, mais néanmoins normaux [nondisordered]. Seul ce juste milieu peut fournir une base pour la critique et l'amélioration des critères diagnostiques actuels.

Je présente ici un aperçu d'une telle analyse, celle du concept de trouble mental en tant que "dysfonction préjudiciable » [harmful dysfunction] que j'ai développé et défendu ailleurs (Wakefield, 1992a, 1992b, 1993, 1999a, 1999b). J'illustre également les implications de l'analyse pour la critique des critères du diagnostic de trouble mental du DSM.

Parmi les analyses du " trouble mental ", il faut établir une division fondamentale entre les approches évaluative et scientifique. Comme Kendell (1986) le mentionne : " la question la plus fondamentale, et également la plus controversée, est de savoir si le trouble et la maladie sont des concepts évaluatifs fondés sur des jugements de valeur, ou bien s'ils sont des termes scientifiques objectifs; en d'autres termes, s'ils sont des termes biomédicaux ou 
sociopolitiques" (p. 25). Je propose une explication hybride selon laquelle le trouble est une dysfonction préjudiciable, où " préjudiciable » est un terme évaluatif se rapportant aux conditions jugées négatives selon les standards socioculturels, et où "dysfonction » est un terme scientifique factuel basé sur la biologie évolutionniste, qui se rapporte à la défaillance d'un mécanisme interne dans l'accomplissement d'une de ses fonctions naturelles.

Puisque notre analyse concerne en définitive le concept général de trouble tel qu'appliqué aux conditions tant physiques que mentales, des exemples dans ces domaines sont utilisés pour la tester. J'utilise " mécanisme interne » comme terme général pour faire référence tant aux structures et organes physiques qu'aux structures et dispositions mentales, tels que les mécanismes motivationnels, cognitifs, affectifs et perceptuels. Certains auteurs distinguent "trouble » [disorder] et " maladie »[disease, illness]; je préfère le terme de " trouble ", plus large, qui recouvre autant les atteintes traumatiques que les maladies, et qui est ainsi plus près du concept général de la pathologie médicale.

Les présuppositions de l'analyse sont les suivantes :

1. L'objectif est de rendre compte des jugements classificatoires largement partagés quant à ce qui est ou non considéré comme des cas évidents de trouble. Les cas controversés sont dans un premier temps mis de côté : ces derniers peuvent être le résultat des limites de la connaissance actuelle, des limites du concept de trouble luimême ou de défis particuliers à l'application de "trouble » au domaine mental.

2. Les membres d'une culture partagent généralement les représentations mentales à l'origine des concepts qui donnent leur signification aux termes linguistiques communs. Ainsi, tout comme le linguiste qui tente de formuler une théorie de la grammaire d'une langue en considérant les jugements communs des individus sur les phrases qui sont et ne sont pas grammaticales, l'analyste des concepts tente de formuler une théorie des représentations conceptuelles communes en considérant les jugements classificatoires communs des membres de la communauté sur ce qui est subsumé ou non sous un concept. Notez qu'on ne pense pas que les jugements classificatoires largement partagés sont acceptables; formuler un concept et juger si une chose donnée est subsumée ou non sous ce concept sont deux choses différentes, et l'objectif est de rendre compte de tels jugements, qu'ils soient acceptables ou non.

3. Je ne présuppose pas qu'il existe une frontière précise ou nette entre les concepts de trouble et d'état normal [nondisorder]. Comme pour la plupart des concepts, je présume que celui de "trouble mental » contient des zones d'indétermination, d'ambiguïté, de 
flou et d'imprécision, et qu'une analyse réussie devrait refléter et rendre compte de tels aspects de nos jugements.

4. Le sens de "trouble » qui s'applique aux troubles mentaux est le même que celui qui s'applique aux troubles physiques. Ce point est crucial si l'analyse vise à confronter les affirmations antipsychiatriques selon lesquelles les troubles mentaux ne sont pas des troubles médicaux. Notez que même si les troubles mentaux sont des troubles médicaux au sens conceptuel, cela ne signifie pas que les troubles mentaux doivent être des troubles psychologiques du cerveau ; les fonctions mentales peuvent être altérées à cause de problèmes représentationnels du " programme " plutôt qu'à cause de problèmes physiologiques du « matériel ».

5. Je me concentre sur la question de ce qui fait d'une condition mentale un trouble mental. Je ne me penche pas sur la façon de délimiter les troubles mentaux par rapport aux troubles physiques. Je laisse également de côté la question complexe de savoir ce qui fait qu'une condition est dite mentale plutôt que physique. Pour mon propos, il suffit de dire que les processus mentaux sont tout bonnement des choses comme les émotions, la pensée, la perception, la motivation, le langage, l'action intentionnelle et d'autres processus semblables. Je ne soutiens donc aucune hypothèse cartésienne quant à un statut ontologique spécial du mental ; il n'est question que d'un ensemble particulier de fonctions et de processus. Je ne m'attarderai pas au principe selon lequel les fonctions sont classées comme étant mentales, mais cela a probablement quelque chose à voir avec ce que les philosophes et les spécialistes des sciences cognitives appellent l'intentionnalité ou la représentationnalité.

6. Je suppose que le concept de trouble est partagé par les profanes et les professionnels, et j'évalue les exemples en gardant cela à l'esprit. Cette supposition va à l'encontre des querelles récentes selon lesquelles "trouble mental» est un concept purement technique défini seulement par un discours professionnel impénétrable (ex. : Kirmayer et Young, 1999).

7. Je pense que, contrairement à certaines conceptions holistes, les concepts ne sont pas identiques à des croyances ou à des théories sur les choses subsumées (Wakefield, 1994a). Par exemple, deux personnes peuvent s'accorder parfaitement sur le concept de " célibataire " mais quitter une réunion avec des croyances entièrement différentes concernant le célibat de certaines personnes parce qu'elles ont des théories différentes quant aux indices révélateurs du célibat (par exemple, l'une croit que l'absence d'un anneau de mariage est un bon signe du célibat, tandis que l'autre ne le croit pas, etc.). 
En voilà assez pour ce qui est des suppositions d'arrière-plan. Je me penche maintenant sur l'analyse de la notion de " trouble».

\section{La composante évaluative du « trouble »}

Comme le suggèrent les explications évaluatives traditionnelles, une condition est un trouble mental uniquement si elle est préjudiciable au regard de valeurs sociales et, de ce fait, si elle justifie potentiellement l'attention médicale. "Préjudiciable » est interprété ici de façon large afin d'inclure toutes les conditions négatives.

Les jugements classificatoires tant profanes que professionnels démontrent que le concept de trouble mental renferme une composante évaluative. Par exemple, l'incapacité d'apprendre à lire qu'entraîne une dysfonction dans le corps calleux (en supposant que cette théorie sur certains types de dyslexie soit vraie) est préjudiciable dans les sociétés alphabétisées mais ne l'est pas dans les sociétés pré-littéraires où lire n'est pas une habileté enseignée ou valorisée ; elle n'est donc pas un trouble dans ces sociétés. Par ailleurs, la plupart des gens ont ce que les médecins appellent des " anomalies bénignes ", c'est-à-dire des malformations mineures qui sont le résultat d'erreurs génétiques ou dans le développement, mais qui n'occasionnent pas de problèmes importants. De telles anomalies ne sont généralement pas considérées comme des troubles. Par exemple, une certaine proportion des spermatozoïdes sont clairement difformes et dysfonctionnels, mais ces dysfonctions internes ne sont pas considérées comme des troubles sauf quand elles occasionnent un préjudice affectant la fonction reproductive. De la même façon, les angiomes bénins sont des petits vaisseaux sanguins dont la croissance s'est emballée, les menant à se raccorder à la peau. Mais, parce qu'ils ne sont pas préjudiciables, ils ne sont pas considérés comme des troubles. L'exigence qu'il y ait préjudice explique également pourquoi le simple albinisme, l'inversion de la position du cœur ainsi que les orteils collés ne sont généralement pas considérés comme des troubles, bien que chacun résulte d'une défectuosité dans la façon dont un mécanisme est censé fonctionner.

J'ai argumenté ailleurs, en défendant la composante évaluative du «trouble », contre l' " erreur essentialiste » scientiste qui perçoit le « trouble mental » comme un concept scientifique ou purement et simplement factuel (Wakefield, 1997b). Mes critiques évaluativistes ont même occasionnellement reconnu que j'ai contribué à nous débarasser de la conception purement factuelle du "trouble" (Sadler, 1999). Le débat persiste quant à la question de savoir si le concept de " trouble mental » est purement évaluatif ou s'il contient une composante factuelle déterminante permettant de distinguer un domaine potentiel de conditions négatives qui sont des troubles, du domaine de celles qui n'en sont pas.

\section{Pourquoi le « trouble » doit-il comporter une composante factuelle ?}

Certaines analyses du concept de «trouble » s'arrêtent ici, soutenant qu'un trouble mental est simplement une condition socialement réprouvée (par 
exemple, Houts, 2001 ; Sedgwick, 1982). Mais il y a de bonnes raisons d'insister sur le fait que la notion de "trouble mental » ne peut pas simplement être un concept évaluatif (Wakefield, 1995 ; 2003). Nous avons besoin d'une composante factuelle additionnelle pour distinguer les troubles des nombreuses autres conditions mentales négatives qui ne sont pas considérées comme des troubles mentaux, telles que l'ignorance, le manque d'adresse, le manque de talent, une faible intelligence, l'illettrisme, la criminalité, les mauvaises manières, la bêtise et la faiblesse morale. Les « troubles mentaux » sont seulement un sous-ensemble des nombreuses choses négatives qui peuvent arriver à une personne.

En effet, parmi des conditions négatives pourtant très similaires, les professionnels tout aussi bien que les profanes distinguent les troubles des états normaux. Par exemple, l'illettrisme n'est pas comme tel considéré comme un trouble, même s'il est déprécié et préjudiciable dans notre société ; toutefois, une condition similaire, dont on croit qu'elle est attribuable à l'incapacité d'apprendre à lire entraînée par une dysfonction neurologique ou psychologique interne, sera, elle, considérée comme un trouble. L'agressivité masculine plus grande et la propension plus grande de l'homme à l'infidélité sont considérées comme négatives mais ne sont généralement pas considérées comme des troubles, parce qu'elles sont perçues comme étant le résultat du fonctionnement naturellement sélectionné du sexe masculin, et ce, malgré que des conditions motivationnelles compulsives similaires soient, elles, perçues comme des troubles. Une explication purement évaluative du "trouble » ne rend pas compte de telles distinctions entre des conditions négatives similaires.

De plus, nous réglons souvent nos conceptions du trouble sur la base de données transculturelles qui peuvent aller à l'encontre de nos valeurs. Par exemple, notre culture ne valorise pas la polygamie, mais nous jugeons qu'il ne s'agit pas d'une défaillance du fonctionnement naturel, donc elle n'est pas considérée comme pathologique, et ce, en partie sur la base de données transculturelles.

Pour des raisons de ce type, la recherche d'une analyse purement évaluative du concept de " trouble » n'a pas su mener à une définition adéquate, du point de vue explicatif, pour distinguer les troubles des autres conditions négatives. L'échec de telles explications neutres à l'égard des faits [fact-neutral] nous pose le défi de proposer une composante factuelle qui puisse expliquer les jugements portant sur les troubles. L'analyse $\mathrm{DP}^{1}$ prétend que la composante factuelle est un des sens du terme dysfonction, que Klein (1978) appelle la «dysfonction de la composante ». Je prétends que cet élément de dysfonction renvoie au jugement faillible, purement non évaluatif, factuel, selon lequel un quelconque aspect du fonctionnement naturel d'un humain fait défaut. La principale objection à cette approche a été de prétendre que le terme

1. Note du traducteur: je traduis «harmful dysfunction» par «dysfonction préjudiciable "; l'acronyme " $\mathrm{DP}$ » remplace donc « $\mathrm{HD}$ » dans le texte original. 
«dysfonction " est lui-même un concept évaluatif. Le défi est donc d'en élucider le sens factuel.

La discussion de ces questions ne tient souvent pas compte de la distinction entre les deux affirmations suivantes : 1 ) les verdicts de dysfonction sont souvent influencés par, associés à, ou déterminés par, des jugements de valeur ; 2) le concept de trouble (y compris la dysfonction) est intrinsèquement (sur le plan sémantique) un concept purement évaluatif. Quand je dis que "dysfonction" (au sens qui nous occupe) est un concept factuel, je rejette seulement la thèse 2 , selon laquelle la signification de "dysfonction " est (au moins partiellement) évaluative. J'accepte la thèse 1 comme étant vraie. Il ressort clairement des comptes rendus historiques et anthropologiques que les valeurs, les normes et les idéologies influencent profondément ce que les gens croient être des fonctions naturelles, en particulier lorsque la compréhension scientifique de ce qui est fonctionnel et dysfonctionnel fait défaut (comme c'est actuellement le cas pour de nombreux aspects mentaux). Mais il n'y a rien là qui implique que le contenu sémantique du concept de " fonction naturelle » soit évaluatif. De façon similaire, si les valeurs religieuses d'un tel l'amène à nier que la Terre tourne autour du Soleil et à affirmer que la Terre a moins de 7000 ans, cela ne veut pas dire que les concepts " tourne autour du Soleil » ou " a moins de 7000 ans » soient des concepts à connotation évaluative [value-laden]. Ce sont des concepts purement factuels, descriptifs, vrais ou faux au sujet de notre planète, indépendants des valeurs de quiconque, même si les croyances d'une personne sur la pertinence de les appliquer à la Terre sont profondément ou même entièrement déterminées par des valeurs.

\section{Les fonctions en tant qu'effets qui expliquent leurs causes}

Le point de vue selon lequel le concept de trouble implique de quelque façon les concepts de fonction et de dysfonction ressort avec une homogénéité remarquable des remarques de nombreux auteurs dont les approches divergent par ailleurs (par exemple, Ausubel, 1971 ; Boorse, 1975 ; 1976a ; 1977 ; Caplan, 1981 ; Kendell, 1975 ; 1986 ; Klein, 1978 ; Macklin, 1981 ; Moore, 1978 ; Ruse, 1973). Spitzer et Endicott (1978) notent l'apparente nécessité et la quasi-universalité de l'usage de « dysfonction » pour donner un sens au concept de «trouble » : "Notre approche rend explicite une supposition sousjacente qui est présente dans tous les débats portant sur la maladie ou le trouble, c'est-à-dire le concept de dysfonction de l'organisme » (p. 37). Ce dont nous avons besoin est donc une analyse satisfaisante des concepts de "fonction » et de « dysfonction. "

Qu'est-ce alors qu'une dysfonction ? Une façon d'aborder le problème serait de penser qu'une dysfonction implique le défaut d'une fonction, autrement dit, l'incapacité pour un mécanisme de l'organisme d'accomplir sa fonction. Cependant, tous les usages de "fonction » et de " dysfonction » ne sont pas pertinents ici. Le sens médicalement pertinent de "dysfonction » n'est évidemment pas le sens usuel du terme, qui se rapporte à l'incapacité d'un 
individu à bien se comporter dans un rôle social ou un environnement donné, comme on le retrouve dans des affirmations telles que "Je vis une relation dysfonctionnelle » ou " Le malaise à l'égard des structures de pouvoir hiérarchiques est dysfonctionnel dans l'environnement des entreprises d'aujourd'hui. » Ces types de problèmes n'ont pas besoin d'être des troubles à proprement parler. De plus, les types de fonctions qui nous occupent ne sont pas ceux qui résultent de décisions sociales ou personnelles d'utiliser une partie de l'esprit ou du corps d'une façon particulière. Par exemple, le nez peut avoir la fonction de soutenir les lunettes, et le cœur accomplit une fonction utile dans le diagnostic médical. Mais la personne dont le nez a une forme telle qu'il ne soutient pas les lunettes n'a pas pour autant un trouble nasal, de même que la personne dont le cœur n'émet pas le son habituel ne souffre pas pour autant d'un trouble cardiaque. Un trouble est différent de l'incapacité à agir d'une manière socialement ou personnellement particulière, précisément parce qu'une dysfonction existe seulement lorsqu'un organe ne peut fonctionner comme il est naturellement censé le faire (c'est-à-dire, indépendamment des intentions humaines). On peut donc présumer que les fonctions qui sont pertinentes ici sont les "fonctions naturelles ", concept au sujet duquel on trouve une abondante littérature, qui sera exploitée ci-dessous (Boorse, 1976b ; Cummins, 1975 ; Elster, 1983 ; Hempel, 1965 ; Klein, 1978 ; Moore, 1978 ; Nagel, 1979 ; Woodfield, 1976 ; Wright, 1973 ; 1976). De telles fonctions sont fréquemment attribuées à de présumés mécanismes mentaux sousjacents ; par exemple, une fonction naturelle de l'appareil perceptuel est de transmettre de l'information plus ou moins précise sur l'environnement immédiat, de sorte que des hallucinations évidentes indiquent une dysfonction ; certains mécanismes cognitifs ont aussi pour fonction de fournir à la personne le degré de rationalité nécessaire aux raisonnements déductifs, inductifs et instrumentaux, de telle sorte qu'il y a dysfonction lorsque la capacité d'effectuer de tels raisonnements s'effondre, comme dans les états psychotiques graves. Hempel (1965) a posé le problème de la « fonction naturelle » comme suit : chaque organe a plusieurs effets, dont la plupart ne sont pas ses fonctions naturelles. Par exemple, le cœur a comme effets de pomper le sang et de produire un son dans la poitrine, mais seul le pompage du sang est une fonction naturelle. Une analyse de la " fonction naturelle " doit spécifier ce qui distingue les fonctions naturelles d'un organe de ses autres effets.

Le concept de fonction s'applique également aux artéfacts, tels que les automobiles, les chaises et les stylos. Il semble plausible que le concept de " fonction " ait été étendu, par analogie, des artéfacts aux organes (Wright, 1973 ; 1976). Par conséquent, l'usage du terme " fonction » dans le cas des mécanismes naturels doit être une façon de faire référence aux propriétés que de tels mécanismes partagent avec les artéfacts. Or la fonction d'un artéfact est simplement l'usage pour lequel il a été conçu ; par exemple, la fonction des automobiles, des chaises et des stylos est, respectivement, de nous permettre de nous déplacer, de nous asseoir et d'écrire, parce que ce sont précisément 
les attributs de ces objets qui expliquent leur existence. Cependant, les organismes et leurs organes se trouvent dans la nature et n'ont pas été véritablement « conçus " par quiconque avec un but précis en tête, donc la conception et la finalité ne peuvent pas être leur propriété commune. Bien sûr, les biologistes évolutionnistes parlent couramment de finalité et de conception lorsqu'ils discutent les fonctions naturelles, mais cela ne fait que reporter le problème ; qu'est-ce qui justifie ce propos métaphorique dans le cas des mécanismes naturels ? L'extension du terme "fonction » des artéfacts aux mécanismes naturels doit être justifiée par une autre propriété commune sous-jacente aux termes " conception » et " finalité ", et pertinente à ces notions.

La fonction d'un artéfact est importante principalement parce que sa valeur explicative est remarquable quant à son rapport avec la conception et la finalité. La fonction explique pourquoi l'artéfact a été conçu, pourquoi il est structuré de telle façon, pourquoi les parties interagissent de telle façon et pourquoi il est utile à certaines choses. Par exemple, nous pouvons partiellement expliquer pourquoi les automobiles existent, pourquoi les moteurs d'automobile sont structurés comme ils le sont, et pourquoi, avec l'apprentissage approprié, on peut se rendre d'un endroit à un autre en automobile, tout cela en se référant simplement à la fonction de l'automobile, qui est d'être un moyen de transport.

Les explications fonctionnelles des artéfacts ont ceci de surprenant qu'un effet (par exemple, le transport) est censé rendre compte d'une certaine manière de l'artéfact même qui produit l'effet (par exemple, l'automobile). Par conséquent, il a quelquefois été soutenu que les explications fonctionnelles dérogent au principe de base de la précédence d'une cause sur son effet. Toutefois, une description de la fonction peut légitimement entrer dans l'explication de l'artéfact s'il existe une théorie additionnelle montrant que les effets mentionnés jouent un rôle dans les événements qui ont précédé sa création. Pour ce qui est des artéfacts, cette théorie est bien connue : le bénéfice "précède » l'artéfact au sens où il est représenté à l'avance dans l'esprit de la personne qui le conçoit. Ainsi, une explication fonctionnelle (par exemple, « la fonction des automobiles est de fournir un moyen de transport » ou, de façon équivalente, "les automobiles existent pour nous fournir un moyen de transport ») est l'ébauche d'une explication causale plus complète : l'artéfact (par exemple, une automobile) existe parce qu'on désirait un certain effet (par exemple, le transport), et on croyait que créer cet artéfact était une façon d'obtenir l'effet ; ainsi, la croyance et le désir qui précédaient l'artéfact ont poussé quelqu'un à créer celui-ci.

J'ai soutenu que la fonction d'un artéfact est importante en vertu de son pouvoir explicatif et que les explications des artéfacts par la fonction prennent diverses formes : l'existence et la structure de l'artéfact sont expliquées par rapport à ses effets. Il y a également d'autres domaines dans lesquels cette forme distincte d'explication justifie l'attribution du terme " fonction ». Par exemple, lorsque les behavioristes parlent d' " analyse fonctionnelle » et de la 
fonction d'un comportement, ils ne font pas uniquement référence aux effets négatifs du comportement. Ils pensent plutôt aux effets du comportement qui renforcent celui-ci et expliquent ainsi son existence, son maintien et sa structure. Encore une fois, c'est la structure de l'explication par les effets [effectexplanatory] qui conduit à parler de fonction.

C'est cette forme d'explication que les affirmations portant sur les artéfacts et les fonctions naturelles ont en commun, et qui justifie l'extension du discours sur la «fonction" des artéfacts aux mécanismes naturels. Par exemple, l'effet de pomper le sang produit par le cœur fait également partie de la définition du cœur, en ce sens que l'on peut légitimement répondre à la question " Pourquoi avons-nous un cœur ? » ou " Pourquoi le cœur existet-il ? » par « Parce que le cœur pompe le sang ». L'effet de pomper le sang entre également dans les explications de la structure et de l'activité détaillées du cœur. Ainsi, pomper le sang est la fonction naturelle du cœur. La recherche anatomique et physiologique est largement consacrée à la spécification des fonctions naturelles des organes et à l'explication de la façon dont les caractéristiques d'un organe participent des fonctions naturelles de celui-ci. Parler de " conception » et de "finalité " dans le cas de mécanismes naturels n'est qu'une façon métaphorique de référer à cette propriété explicative unique selon laquelle l'effet d'un mécanisme rend compte de celui-ci. Donc, la " fonction naturelle " peut être analysée comme suit : la fonction naturelle d'un organe ou d'un autre mécanisme est un effet de l'organe ou du mécanisme qui entre dans l'explication de l'existence, de la structure ou de l'activité de l'organe ou du mécanisme. Une " dysfonction » existe lorsqu'un mécanisme interne est incapable d'accomplir sa fonction naturelle. (En fait, cela est une première approximation; il y a certains problèmes additionnels dans l'analyse du terme « fonction » dont on ne peut s'occuper ici et qui requièrent une analyse plus poussée, comme celle fournie dans Wakefield 1999b ; 2000a)

L'analyse ci-dessus s'applique aussi bien aux fonctions naturelles des mécanismes mentaux qu'aux fonctions des organes physiques et constitue donc une base commune pour l'attribution de troubles physiques et mentaux. Tout comme les artéfacts et les organes, les mécanismes mentaux tels que les mécanismes cognitifs, linguistiques, perceptuels, affectifs et motivationnels ont des effets si extraordinairement bénéfiques et dépendent d'interactions si complexes et si harmonieuses qu'ils ne peuvent être entièrement accidentels. Ainsi, les explications fonctionnelles des mécanismes mentaux sont parfois justifiées par ce que nous savons de la façon dont les gens réussissent à survivre et à se reproduire. Par exemple, la capacité de communiquer est une des fonctions des mécanismes linguistiques, éviter le danger est une fonction de la réaction de peur, de même qu'entraîner le repos et le sommeil est une fonction de la fatigue. Ces explications fonctionnelles mènent au diagnostic de " dysfonction ", lorsque les mécanismes respectifs ne réussissent pas à remplir leurs fonctions, comme dans l'aphasie, la phobie et l'insomnie. 
Une caractéristique importante des explications fonctionnelles est qu'elles peuvent être plausibles et très utiles même lorsqu'on en sait très peu sur la nature véritable d'un mécanisme. Pour ce qui est des mécanismes et des artéfacts, les effets qu'ils produisent sont si remarquables et dépendent d'interactions si harmonieuses et si compliquées qu'en l'absence d'hypothèse de rechange il est souvent raisonnable d'émettre l'hypothèse que l'effet n'est pas accidentel, en ce sens que l'artéfact existe parce qu'il a ces effets. Par exemple, le fait que l'œil nous permet de voir, que les jambes nous permettent de marcher ou que le cœur pompe le sang, ou encore que l'automobile nous fournit un moyen de transport ne peut être simplement un heureux hasard. Les yeux existent donc, entre autres raisons, parce qu'ils nous permettent de voir, autrement dit, le fait que les yeux assurent la vision doit entrer de quelque façon dans l'explication de la raison pour laquelle nous avons des yeux. Cela fait de la vue une fonction de l'œil. Évidemment, on peut se tromper dans de telles tentatives d'explication; ce qui semble non accidentel peut se révéler accidentel. Cependant, nous sommes souvent dans le vrai, et les théories explicatives des fonctions transmettent un savoir complexe qui ne peut être aussi efficacement et facilement transmis d'aucune autre façon. Ainsi, bien qu'aujourd'hui nous soyons dans une large mesure ignorants des détails des mécanismes mentaux et de leurs fonctions comme de leurs dysfonctions, nous pouvons souvent vraisemblablement reconnaître qu'une dysfonction se produit sur la base de preuves circonstancielles. La notion de « conception » se rapporte à la notion explicative selon laquelle certains effets sont responsables de l'existence et de la structure même des mécanismes qui produisent ces effets.

\section{Dysfonction et théorie de l'évolution}

Comme dans le cas des artéfacts, les explications de la fonction naturelle semblent en surface enfreindre le principe de la précédence de la cause sur ses effets. Par exemple, "le désir sexuel existe parce qu'il amène les gens à s'accoupler et à se reproduire " semble expliquer le désir sexuel comme une chose qui normalement survient après lui. Comprendre exactement comment et dans quel sens de tels effets peuvent jouer un rôle en déclenchant les mécanismes appropriés requiert une théorie additionnelle.

Dans le cas des artéfacts, c'est une représentation mentale préalable de l'effet qui explique l'existence de l'artéfact. La recherche d'une explication causale démystifiante similaire pour le cas des fonctions naturelles a donné lieu à une énigme qui persiste depuis des siècles. Jusqu'à tout récemment, nous ne pouvions faire face à l'énigme qu'en supposant l'existence d'un Dieu qui a intentionnellement créé nos mécanismes internes suivant de bienveillantes intentions, ce qui réduisait ainsi les fonctions naturelles à un cas spécial de fonctions des artéfacts.

Aujourd'hui, la théorie de l'évolution nous fournit une meilleure explication de la façon dont les effets d'un mécanisme peuvent expliquer la 
présence et la structure de celui-ci. En bref, voici comment : les mécanismes dont les effets ont contribué au succès des organismes en matière de reproduction sur un nombre suffisant de générations ont augmenté en fréquence, ont donc été "sélectionnés naturellement ", et se retrouvent dans les organismes actuels. Donc, expliquer un mécanisme par ses fonctions naturelles peut être considéré comme une façon détournée de faire référence aux explications causales en termes de sélection naturelle. Comme la sélection naturelle est le seul moyen connu par lequel un effet peut expliquer le mécanisme naturel qui le produit, les explications évolutionnistes justifient sans doute toutes les attributions correctes des fonctions naturelles. En conséquence, une approche évolutionniste du trouble mental (Buss, 1984 ; 1991; Wakefield, 1989a) est essentielle à la compréhension de la psychopathologie.

$\mathrm{La}$ " dysfonction » est ainsi purement et simplement un concept scientifique factuel. Toutefois, découvrir ce qui est en fait naturel ou dysfonctionnel (et ainsi ce qui est pathologique) peut se révéler difficile et susciter une controverse scientifique, surtout en ce qui concerne les mécanismes mentaux dont nous ignorons encore beaucoup de choses. Cette ignorance est en partie responsable de la grande confusion et de la controverse à propos des conditions qui sont véritablement des troubles mentaux. Toutefois, paradoxalement, cette ignorance de l'historique causal et de la nature précise des mécanismes mentaux rend d'autant plus nécessaire le recours à des explications fonctionnelles basées sur des inférences concernant le rôle spécifique des mécanismes mentaux. À cet égard, nous en sommes maintenant à une étape de compréhension comparable d'un certain point de vue à la position des anciens médecins qui devaient recourir à des inférences similaires pour évaluer les troubles physiques. Ainsi, même s'ils ne savaient rien des mécanismes en jeu dans la vision ou de l'histoire naturelle de l'œil, ces médecins n'en comprenaient pas moins, sur la base d'inférences fonctionnelles, que la cécité et d'autres conditions physiques étaient des dysfonctions. Plus nous en saurons sur les fonctions des mécanismes mentaux issus de la sélection naturelle, plus nos jugements sur la dysfonction deviendront sûrs.

Du point de vue de la théorie des concepts, on doit techniquement distinguer l'analyse conceptuelle du trouble, en tant que dysfonction préjudiciable, de la théorie évolutionniste de la dysfonction. Le trouble ne peut être directement analysé d'un point de vue évolutionniste parce que l'analyse vise à saisir un concept médical intuitif et profane largement partagé, lequel existait bien avant que la théorie de l'évolution ne soit formulée et était partagé par plusieurs de ceux qui ignorent ou rejettent la théorie de l'évolution; c'est le cas des chrétiens fondamentalistes, qui croient que Dieu a façonné les mécanismes humains. Les déistes et les athées s'accordent quant aux conditions qui sont des dysfonctions et des troubles parce que l' "explication par la conception » [explanatory-design notion of function] sous-jacente dans la notion de fonction les satisfait, et ils utilisent la preuve circonstancielle pour appliquer ce concept plus ou moins de la même manière, en dépit de leurs théories 
radicalement différentes sur la façon dont de tels mécanismes « conçus pour une fin » sont apparus. Donc, on n'a pas à comprendre ou à accepter la théorie de l'évolution pour être en possession du concept de trouble. C'est une découverte scientifique capitale, et non une question de définition, que la sélection naturelle est le processus essentiel qui explique les fonctions et les dysfonctions. "Trouble » signifie " dysfonction préjudiciable ", et l'évolution est la théorie la plus convaincante de la nature des fonctions et des dysfonctions.

Ainsi, un trouble n'existe que lorsqu'un mécanisme interne est dysfonctionnel, en particulier en ce sens qu'il est incapable d'accomplir l'une de ses fonctions naturelles (à ce stade de l'analyse, la fonction naturelle est entendue dans le sens intuitif qui a été utilisé depuis des millénaires, non dans un sens évolutionniste technique). La notion apparemment anthropomorphique de la fonction d'un mécanisme biologique est analysée en des termes résolument scientifiques, causaux, pour indiquer que certains effets des mécanismes biologiques sont si complexes, avantageux et délicatement organisés qu'ils ne peuvent être des répercussions accidentelles de processus causaux aléatoires. Comme les fonctions intentionnellement déterminées des artéfacts, ils doivent plutôt, de quelque façon, faire partie de l'explication de la raison pour laquelle les mécanismes sous-jacents existent et sont structurés comme ils le sont. Par analogie avec les artéfacts, ces fonctions sont souvent censées correspondre à ce pour quoi les mécanismes sont " conçus ". Les affirmations selon lesquelles certains effets d'un mécanisme sont utiles n'offrent aucune explication du mécanisme ; l'utilité ne peut être attribuable à la chance. Au contraire, les attributions de fonction ont une prétention explicative implicite, à savoir que l'utilité explique en partie le mécanisme.

Strictement parlant, l'analyse explicative de la fonction naturelle complète l'analyse conceptuelle du trouble. Toutefois, cette analyse n'explique pas comment un effet (par exemple, le pompage ou la vision) pourrait expliquer sa propre cause (le cœur ou les yeux), pas plus que cette analyse ne nous fournit de critère par lequel on pourrait scientifiquement distinguer les fonctions naturelles des autres effets d'une façon plus précise que celle permise par l'intuition du sens commun. L'analyse mène inévitablement à la question suivante : quel genre de processus sous-jacents sont possiblement responsables de ce qui semble être conçu intentionnellement dans les systèmes naturels, sans qu'il n'y ait pourtant aucun concepteur ? Pour répondre à cette question, il faut une théorie scientifique de la façon dont de tels effets explicatifs peuvent se produire. La tentative de répondre à cette question mène à l'étape suivante dans l'argument ci-contre : la théorie évolutionniste fournit la seule explication scientifique plausible qui existe actuellement de la façon dont les fonctions normales d'un mécanisme peuvent expliquer l'existence et la structure de celui-ci (les explications antérieures du même phénomène incluaient les " causes finales » d'Aristote et « la volonté divine » de Thomas d'Aquin). Selon la théorie évolutionniste, certains effets d'un mécanisme étaient avantageux pour les organismes primitifs et ont de ce fait entraîné la sélection naturelle 
de celui-ci. En conséquence, ils font partie d'une explication de l'existence et de la structure du mécanisme dans les organismes actuels. Ces effets naturellement sélectionnés sont les fonctions normales des mécanismes correspondants. Cet argument théorique mène à la conclusion que les troubles sont des défaillances des mécanismes quant à l'exécution de fonctions pour lesquelles ils ont été sélectionnés naturellement.

On pourrait objecter que l'élément dysfonctionnel dans les troubles est parfois une fonction sociale qui n'a rien à voir avec des catégories naturelles et universelles. Par exemple, les troubles de lecture semblent indiquer le défaut d'une fonction sociale, parce qu'il n'y a rien de naturel ou de déterminé à l'avance dans la lecture. Cependant, l'analphabétisme engendre le même genre de problèmes que les troubles de lecture, et pourtant on ne le considère pas comme un trouble. L'incapacité de lire est considérée comme un signe de trouble seulement quand les circonstances suggèrent que la raison de cette incapacité se situe dans la défaillance d'un certain mécanisme du cerveau à exécuter sa fonction normale. Il y a beaucoup d'écarts chez les individus, dans l'accomplissement de leurs fonctions sociales, et ils ne sont pas considérés comme des troubles tant qu'ils ne sont pas attribués à une fonction normale défaillante.

Si l'on porte attention à la liste des troubles du Diagnostic and Statistical Manual of Mental Disorders (DSM) de l'Association américaine de psychiatrie (1994), il est clair qu'en général nous avons affaire à une liste des diverses manières dont quelque chose peut faire défaut au regard des caractéristiques apparemment déterminées de l'esprit. Très grossièrement, les troubles psychotiques indiquent que les processus de la pensée sont incapables de faire ce pour quoi ils ont été conçus. Les troubles d'anxiété résultent d'un défaut des mécanismes responsables de la peur et de l'anxiété qui empêchent ceux-ci de fonctionner de la façon dont ils ont été conçus, les troubles de dépression indiquent que des mécanismes régulateurs de la tristesse et de réaction à la perte [lossresponse] sont défectueux, les troubles de comportement perturbateur chez les enfants résultent de la défaillance des processus de socialisation et de ceux qui sont en jeu dans la conscience et la coopération, les troubles du sommeil résultent du mauvais fonctionnement des mécanismes du sommeil, les dysfonctions sexuelles résultent des défectuosités de nombreux mécanismes en jeu dans la motivation et la réponse sexuelle, les troubles nutritifs sont causés par la défectuosité des mécanismes suscitant l'appétit, et ainsi de suite. Il y a également une certaine part d'absurdité dans le manuel. Toutefois, la très grande majorité des catégories sont reliées à des maladies que même un profane pourrait correctement reconnaître comme des défectuosités dans le fonctionnement de mécanismes conçus pour une certaine finalité.

Lorsque nous établissons une distinction entre le simple chagrin et la dépression pathologique, entre le comportement délinquant épisodique et un trouble de conduite, entre la simple criminalité et le trouble antisocial de la personnalité, entre la tristesse occasionnelle et un trouble d'adaptation, entre 
l'analphabétisme et un trouble de lecture, entre le manque d'empathie d'un groupe envers ses ennemis et l'absence d'empathie envers tous chez un sociopathe, entre l'exubérance infantile courante et le déficit d'attention d'un hyperactif, nous utilisons implicitement le critère de " défectuosité de la fonction à exécuter ce pourquoi elle a été conçue ». Toutes ces conditions normales ou anormales - sont dévalorisées et préjudiciables, et leurs effets peuvent être très semblables sur le plan comportemental. Pourtant, certaines sont considérées comme pathologiques et d'autres pas. Le critère de la fonction naturelle explique de telles distinctions.

Ce critère spécifie que même les états biologiques préjudiciables dans l'environnement habituel ne sont pas considérés comme des troubles s'ils sont perçus comme des caractéristiques de mécanismes conçus en vue d'une certaine fin. Par exemple, un goût prédominant pour les matières grasses n'est pas considéré comme un trouble parce qu'il est considéré comme un trait naturel qui nous fut utile pour obtenir les calories nécessaires en des temps où l'accès à la nourriture était limité, même s'il peut nous tuer dans le contexte actuel d'abondance de nourriture. La moyenne plus élevée d'agressivité chez les hommes n'est pas considérée comme un trouble général des hommes parce qu'elle est perçue comme la façon d'être de ceux-ci, même si dans la société d'aujourd'hui elle est sans doute préjudiciable. Les féministes prétendent parfois que les hommes souffrent d'un trouble général d'intoxication à la testostérone, mais cela est habituellement tenu pour une plaisanterie et non pour un jugement classificatoire sérieux (bien sûr, il existe des troubles de l'agressivité ; ici comme ailleurs, le caractère naturel des gens peut s'exprimer de façon pathologique.)

En somme, un trouble mental est une dysfonction mentale préjudiciable. Si l'analyse de la dysfonction préjudiciable est adéquate, alors les catégories du trouble mental façonnées par la société nous offrent deux éléments d'information. Premièrement, elles dénotent un jugement de valeur sur l'état que la société considère comme étant négatif ou préjudiciable. Deuxièmement, elles affirment le fait que le mal est dû à la défaillance d'un fonctionnement déterminé de l'esprit ; cette affirmation peut être vraie ou fausse, mais en tout cas elle révèle ce que la société pense être le fonctionnement naturel de l'esprit humain ou celui pour lequel il a été conçu.

\section{Qu'advient-il de Foucault ?}

La faiblesse des points de vue évaluatifs ou sceptiques du trouble mental, qui refusent l'objectivité de la "dysfonction ", a trait à l'absence de raisons pour lesquelles on pourrait élaborer une critique des critères diagnostiques de la psychiatrie. Si tous les critères sont aussi dénués de fondement, il n'y a aucun espoir de les améliorer pour les rendre valides. Un ensemble donné de critères est tout aussi dénué de fondement que n'importe quel autre. Ce n'est pas ainsi que la majorité d'entre nous envisageons la situation. Par exemple, nous pensons que les médecins conservateurs de l'époque victorienne étaient tout 
simplement dans l'erreur lorsqu'ils classaient la masturbation et l'orgasme clitoridien comme des troubles, que les médecins sudistes d'avant-guerre étaient tout simplement dans l'erreur en classant les esclaves fugitifs comme souffrant d'automatisme ambulatoire, et que Freud était tout simplement dans l'erreur en considérant les femmes à qui l'insaisissable orgasme vaginal faisait défaut comme étant perturbées. Toutefois, si les normativistes ont raison, on doit dire que relativement aux valeurs qui étaient alors en vigueur parmi ceux qui les pratiquaient, il s'agissait de troubles authentiques et de diagnostics parfaitement adéquats. Ou bien, si les antipsychiatres ont raison, alors on doit dire que ces diagnostics n'étaient pas plus erronés que nos diagnostics sur la schizophrénie, les troubles de panique ou maniaco-dépressifs. D'une façon ou d'une autre, les distinctions dont on a besoin ne peuvent pas être faites.

L'objectivité de la « dysfonction » permet de faire de telles distinctions et d'expliquer pourquoi de nombreuses pratiques diagnostiques sont incorrectes - nommément, parce qu'elles ne réussissent pas à distinguer des autres les conditions occasionnées par des dysfonctions. L'objectivité de la dysfonction limite également la relativité culturelle du " trouble ». Les dysfonctions définissent les troubles possibles et les valeurs culturelles déterminent quelles dysfonctions sont considérées comme des troubles. Les troubles ne peuvent pas être entièrement définis à partir des valeurs.

Seule une théorie de la nature humaine peut constituer la base adéquate pour une critique sociale convaincante, car un exemple convaincant de la déliquescence des structures sociales ne peut être avancé que par rapport aux besoins dictés par la nature humaine. Les difficultés résultant du relativisme de Foucault, qui a enfreint ce principe, sont bien connues et résumées dans cette phrase : "Sur quoi Foucault peut-il prendre appui ? ». C'est-à-dire, si tout est fondé sur l'exercice du pouvoir - y compris, par exemple, le sens de la justice et même la préférence du corps pour le plaisir sur la douleur - sur quelle autre base pouvons-nous alors nous appuyer pour soutenir qu'une organisation sociale quelconque est meilleure qu'une autre ? Dans le domaine de la psychopathologie, il semble que l'analyse du concept de trouble mental, et la reconnaissance du fait qu'il existe vraiment des dysfonctions mentales au sens littéral de défectuosités des fonctions naturelles déterminées nous donne un point d'appui et nous soulage de l'exigence d'établir les distinctions requises.

Cela signifie-t-il que l'analyse foucaldienne selon laquelle le pouvoir est inscrit dans les discours doit être entièrement rejetée ? Pas du tout. Au contraire, une critique foucaldienne des sources du pouvoir et des implications de ce concept doit être intégrée à une analyse conceptuelle préalable, élaborée à partir de celui-ci et respectant sa nature (Wakefield, 2002b). On doit comprendre un concept avant d'être en mesure de comprendre comment il est utilisé à des fins de contrôle.

L'analyse conceptuelle une fois complétée, deux analyses foucaldiennes connexes de la structure conceptuelle peuvent être entreprises et greffées à l'analyse conceptuelle. Premièrement, il y a la question de savoir pourquoi ce 
concept particulier est saillant à cette époque et dans cette culture. Cette analyse situe le concept dans un contexte socio-historique plus large, offrant une généalogie foucaldienne qui explique comment un concept plutôt qu'un autre devient prédominant et organise la pensée et l'action. Par exemple, on peut se demander pourquoi le concept de trouble mental, plutôt que d'autres concepts connexes, est privilégié quant aux ressources sociales mises à disposition pour fournir de l'aide. En situant l'analyse conceptuelle dans un contexte social plus large, vous obtenez la question stratégique foucaldienne : pourquoi ce concept?

D'un autre côté, la structure conceptuelle elle-même est le contexte initial d'une question tactique foucaldienne plus pointue : comment l'emploi de ce concept dans des jugements classificatoires est-il manipulé à la faveur du pouvoir ? Chaque concept comprend de l'indétermination, de l'ambiguité et des limites floues. Ce qui offre d'amples possibilités de manipulation des jugements classificatoires en vue de tirer avantage du développement d'un concept dans un sens plutôt que dans un autre. Les controverses sur l'application de concepts tels que celui de trouble mental sont possibles parce que les concepts essentialistes, en particulier, sont très abstraits, et il y a un grand nombre d'étapes intermédiaires entre le concept lui-même et les jugements classificatoires concrets, ce qui laisse beaucoup d'espace à la divergence. Il est crucial de comprendre la logique des concepts abstraits sous-jacents dans une analyse de telles controverses, parce que c'est la logique du concept qui explique pourquoi certaines inférences (peut-être fallacieuses) peuvent sembler avoir un sens, tandis que d'autres semblent ne pas en avoir. Autrement dit, le concept aide à expliquer la nature et les possibilités des controverses liées à son utilisation. Ainsi, nous pourrions par exemple nous demander comment de nouvelles catégories de comportements en viennent à être interprétées comme des troubles ou réinterprétées comme des états normaux par le DSM (c'est-à-dire, introduites ou retirées du manuel), et dans quelle mesure la logique du concept de trouble et la preuve de dysfonction, contrairement à d'autres processus sociaux, déterminent de tels changements. Nous pourrions également examiner quelles techniques sont utilisées pour étendre le domaine du concept de " trouble mental " à un plus grand éventail de conditions lorsque les intérêts économiques des fabricants de médicaments et des thérapeutes sont en jeu (comme dans le cas des pressions actuelles en vue de définir la dépression en tant que trouble dans la pratique médicale en général), et ce, sans égard à l'interprétation contextuelle selon laquelle la condition pourrait être due à une perte réelle et ainsi ne pas être pas un trouble du tout.

Les succès des tactiques adoptées en tentant d'employer un concept dépendront de leur capacité à convaincre les autres que son emploi est justifié. La nature et le succès de telles tactiques dépendront de façon très sensible de la logique du concept et de la façon dont ses caractéristiques logiques peuvent être exploitées. C'est donc seulement en intégrant les trois niveaux analytiques mentionnés ci-dessus - c'est-à-dire, en plaçant l'analyse conceptuelle d'une 


\section{Philosophiques / Printemps 2006}

base de données immédiatement accessibles à l'esprit [evidential] entre deux couches d'analyse foucaldienne - qu'une explication sociale complète d'un concept et de son emploi peut être réussie.

\section{Qu'advient-il de Szasz ?}

Les sceptiques expriment de nombreuses inquiétudes d'ordre pratique, éthique et épistémologique quant au concept de trouble mental et à son application dans le diagnostic psychologique. Ils notent, par exemple, que les gens étiquetés comme souffrant de troubles mentaux sont souvent stigmatisés, que le diagnostic psychologique est souvent utilisé à des fins de contrôle social et qu'il est souvent difficile de dire si une personne souffre d'un trouble mental. Toutefois, de telles inquiétudes, aussi légitimes et importantes puissent-elles être, doivent être séparées des questions qui concernent la cohérence et la logique du concept de "trouble» (Gorenstein, 1984 ; Horwitz, 1982, p. 5). La nécessité d'une telle séparation des problèmes peut être mise en lumière par un exemple médical : les personnes identifiées comme séropositives sont souvent stigmatisées socialement ; cet étiquetage est souvent utilisé à des fins de contrôle social, car, en raison des imperfections des tests disponibles, il est souvent difficile d'établir si une personne est séropositive. En dépit de ces problèmes, "séropositif » est une catégorie parfaitement cohérente et la condition de séropositivité existe malheureusement bel et bien. Les problèmes pratiques, éthiques et épistémologiques ne démontrent tout simplement pas que quelque chose est erroné dans le concept de trouble mental.

Un autre type d'argument sceptique est mieux ciblé, car il s'adresse directement à la nature du trouble. Szasz (1974 ; voir aussi Sarbin, 1967 ; 1969) est celui qui présente cet argument de la façon la plus explicite, lequel est également implicite dans plusieurs autres positions sceptiques. Szasz suppose d'abord que " trouble physique » est un concept légitime basé sur un fondement clair, à savoir que le trouble consiste en une lésion physique, où le terme "lésion" se rapporte à une déviation reconnaissable dans la structure anatomique. L'argument se poursuit avec l'observation que le "trouble mental » est l'extension du concept de « trouble physique » dans le domaine psychique. Par conséquent, les troubles mentaux n'existent bel et bien que si le même concept de "trouble " qui s'applique aux conditions physiques s'applique également aux conditions mentales étiquetées "troubles»; autrement l'extension de "trouble » aux conditions mentales est tout au plus une métaphore. Szasz soutient ensuite que le terme de " trouble mental » est utilisé pour étiqueter des comportements hors normes sur le plan social et que le fonctionnement psychologique étiqueté « trouble mental » n'est d'ordinaire accompagné d'aucune lésion identifiable du cerveau ou de quelque autre partie du corps (Szasz suppose implicitement qu'aucune lésion ne sera finalement découverte, qui pourrait expliquer une telle condition.) Ainsi, le concept de " trouble » en tant que "lésion ", valable pour les conditions physiques, n'est pas valable pour les conditions mentales, donc les " troubles mentaux » ne 
sont donc pas à proprement parler des troubles. Szasz conclut ainsi : « il n'existe pas de chose telle que la «maladie mentale»» $(1974$, p. 1). La faiblesse dans l'argumentation de Szasz réside dans l'inexactitude de l'explication du trouble physique en tant que "lésion ». L'explication se compose de deux thèses : qu'une lésion (ou une structure corporelle anormale) est une déviation statistique d'une structure anatomique normale et qu'un trouble physique est une lésion. Malheureusement, l'idée qu'une lésion puisse être reconnue directement par sa structure anatomique déviante est erronée. Les structures corporelles varient normalement d'une personne à l'autre, et de nombreuses variations normales sont tout aussi insolites que n'importe quelle lésion. De plus, certaines lésions sont statistiquement non déviantes au sein d'une culture, comme l'artériosclérose, l'irritation pulmonaire mineure ou la gingivite en Occident, ainsi que les ravages de l'ankylostome et de la malaria dans certaines autres cultures. Donc, la reconnaissance d'une lésion n'est pas seulement une question d'observation des déviances anatomiques. Ensuite, et de façon plus importante encore, ce n'est pas l'existence d'une lésion qui définit les « troubles ». D'abord, il y a des troubles physiques pour lesquels il n'y a pas de lésions physiques connues, tels que la névralgie trigéminale ou le prurit sénile (Kendell, 1975). De plus, une lésion peut être une anomalie bénigne qui n'est pas un trouble, comme lorsque le cœur est positionné du côté droit du corps, tout en conservant son intégrité fonctionnelle. Donc, l'explication en tant que "lésion » du " trouble physique » échoue, et, avec elle, l'argument des sceptiques selon lequel le concept de "trouble» ne peut être appliqué comme tel aux conditions mentales.

Comment, alors, reconnaissons-nous les déviations qui sont des lésions et les lésions qui sont des troubles ? Nous reconnaissons une variation dans la structure anatomique comme étant une lésion plutôt qu'une variation normale si la variation diminue la capacité d'une structure particulière à accomplir les fonctions pour lesquelles elle a été conçue. La détérioration d'un mécanisme précis peut être appelée "dysfonction d'une composante" (Lewis, 1967 ; Klein, 1978). C'est pourquoi nous reconnaissons la dysfonction ou la lésion d'une composante comme étant un trouble seulement si la déviation du fonctionnement des parties affecte le bien-être de l'organisme de façon préjudiciable. Par exemple, la raison pour laquelle les orteils collés, l'albinisme et l'inversion de la position du cœur ne sont pas considérés comme des troubles même s'ils constituent des variations anatomiques anormales, c'est qu'ils ne nuisent pas de manière importante à la personne. Ainsi, la notion de "dysfonction préjudiciable » semble expliquer les deux choses que les "lésions » des sceptiques ne peuvent expliquer, à savoir, d'une part, quelles déviations anatomiques sont des lésions, et, d'autre part, quelles lésions constituent des troubles.

$\mathrm{Si}$ « lésion» est avant tout un concept fonctionnel, alors les conditions mentales et physiques peuvent bel et bien être des troubles pour les mêmes raisons, à savoir, à cause de leurs conséquences fonctionnelles (Boorse, 1976a, pp. 62-63). Les processus mentaux jouent des rôles spécifiques à l'espèce, 
importants dans la survie et la reproduction humaine, de sorte qu'il n'y a aucune raison de douter qu'ils ont été sélectionnés et qu'ils ont des fonctions naturelles, comme Darwin lui-même l'a souvent souligné (Boorse, 1976a, p. 64). Grâce à notre héritage évolutif, nous sommes dotés de mécanismes tels qu'un foie et un cœur, et, grâce au même héritage, nous sommes dotés de mécanismes mentaux tels que les nombreuses dispositions et structures cognitives, motivationnelles, affectives, identitaires, hédoniques, linguistiques et comportementales. Certaines conditions mentales diminuent la capacité de ces mécanismes mentaux à accomplir les fonctions pour lesquelles ils ont été conçus. Dans ces cas, il y a une "dysfonction partielle » du mécanisme mental concerné. Le concept de trouble, qu'il soit appliqué aux troubles du foie, aux troubles cardiaques ou aux troubles mentaux, a trait aux dysfonctions partielles qui nuisent à l'individu. Contrairement aux affirmations de Szasz et de Sarbin, la notion de trouble mental n'est pas un mythe fondé sur une navrante métaphore, mais une application fidèle, au domaine mental, du même concept de «dysfonction préjudiciable » qui s'applique au domaine physique.

\section{Critique des critères diagnostiques du DSM}

Je me concentre ici sur l'usage de l'analyse DP afin de critiquer les critères diagnostiques du DSM. Comme les tenants du mouvement antipsychiatrique, je constate une médicalisation excessive des conditions normales. Pour une analyse plus poussée, voir Wakefield et First (2003), et Wakefield (1996 ; 1997a ; 1998 ; 1999c ; 2002a).

Les symptômes sont bien entendu préjudiciables. L'analyse DP suggère que la principale source de faux diagnostics positifs dans le DSM (c'est-à-dire, les conditions qui ne sont pas des troubles authentiques, mais qui sont classées à tort par les critères du DSM comme des troubles) est probablement que les conditions correspondant aux critères diagnostiques ne satisfont pas toujours les critères requis pour qu'il ait "dysfonction ". Souvent, on peut discerner si on a affaire à une dysfonction en replaçant tout simplement le comportement dans son contexte et en examinant si le comportement est une réaction normale ou naturelle à des conditions environnementales, ou bien s'il résulte de mécanismes internes déréglés. Le DSM ne prend généralement pas le contexte en considération dans le diagnostic et se concentre sur les symptômes et les comportements, ôtant ainsi la possibilité de distinguer les conditions pathologiques des conditions normales comportant des manifestations similaires. Je présente ici quelques exemples d'égarements, dans les critères du DSM, dus à l'incapacité de rencontrer les conditions requises pour le concept de "dysfonction":

Le critère du trouble dépressif majeur comporte une exception pour les deuils sans complications (jusqu'à deux mois de symptômes suivant la perte de l'être aimé sont considérés comme normaux), mais aucune exception pour des réactions tout aussi normales à d'autres pertes majeures, telles que le diagnostic médical d'une maladie incurable pour soi-même ou pour l'être 
aimé, la séparation de l'époux ou de l'épouse, la fin d'une aventure amoureuse intense ou la perte d'un emploi et d'un fond de retraite. Les réactions à de telles pertes peuvent correspondre aux critères diagnostiques du DSM mais ne sont pas nécessairement des troubles. Si la réaction d'un individu à une perte comprend seulement, par exemple, deux semaines d'humeur maussade, une perte d'engouement pour les activités normales, l'insomnie, la fatigue et la capacité réduite à se concentrer au travail, alors la réaction de l'individu rencontre les critères du DSM pour ce qui est du trouble dépressif majeur, même si une telle réaction n'est pas nécessairement plus pathologique que dans le cas du deuil. En clair, l'exigence indispensable pour qu'un trouble dépressif soit considéré comme une dysfonction - dans laquelle, peut-être, les mécanismes de réactions à la perte sont disproportionnés et ne réagissent pas de la façon dont ils sont conçus pour le faire - n'est pas rencontrée de façon adéquate par les critères du DSM.

Le trouble de l'adaptation est défini comme une réaction à un facteur de stress identifiable qui : 1) provoque une détresse marquée excédant la réaction anticipée à l'exposition à l'agent de stress ; ou 2) affecte de façon importante le fonctionnement scolaire, professionnel ou social. La première clause concernant la réaction de détresse qui " excède les attentes " conduit le tiers supérieur de la distribution normale de la réactivité au stress à être classifiée comme souffrant d'un trouble et donc, selon toute évidence, elle ne réussit pas à tenir compte de façon adéquate de la variation normale. Elle ne tient pas plus compte des facteurs contextuels faisant qu'une personne a de bonnes raisons pour réagir plus intensément qu'une autre. Le second critère concernant les difficultés à " assumer un rôle » [rôle-impairment] classe au nombre des troubles une réaction normale à l'adversité qui altère temporairement le fonctionnement habituel (par exemple, on ne veut pas socialiser, ou on n'est pas disposé à aller au travail). Pourtant, l'abandon temporaire du rôle fonctionnel habituel est souvent précisément le résultat normal des réactions adaptatives. Ici aussi, les critères contiennent une exception pour le deuil, mais pas pour des réactions tout aussi habituelles face au malheur autres que la mort d'un être cher. En clair, l'essence d'un trouble d'adaptation est que quelque chose n'a pas fonctionné dans les mécanismes d'adaptation, qui sont vraisemblablement conçus pour restituer graduellement à l'individu, peut-être après une période de recul, son état homéostatique, à la suite d'un certain stress ou de certains changements dans les circonstances de sa vie. Cet élément essentiel d'une dysfonction dans les mécanismes d'adaptation n'est pas pris en considération dans l'ensemble des critères du DSM.

La catégorie des états de stress aigu semble contribuer à médicaliser des réactions normales au stress. Si un événement terrifiant, comme la menace de mort, les blessures, le viol, cause la peur, la détresse ou l'horreur (comme il se devrait normalement) et qu'un individu présente des symptômes de réaction au stress (par exemple, se sent étourdi et « distant ", pense à l'événement, réagit aux rappels de l'événement d'une façon qui lui est pénible ou dommageable) 
après plus de deux jours, alors il est considéré comme perturbé. De plus, les critères sont décrits de telle façon que les symptômes dissociatifs les plus extrêmes doivent se présenter uniquement pendant que l'événement est vécu ; ils ne doivent pas se poursuivre par la suite. Après l'événement, on n'a le droit d'être affligé par ses rappels ou de continuer de ruminer là-dessus, de tenter d'éviter ces rappels et de demeurer angoissé et incommodé dans notre fonctionnement pendant plus de deux jours. Il semblerait que de nombreuses réactions saines présentent de telles caractéristiques. Sans aucun doute, certaines réactions aiguës au stress sont suffisamment graves et préjudiciables pour être considérées comme des troubles, mais les critères du DSM ne distinguent pas convenablement ces troubles des réactions habituelles au stress.

Les critères diagnostiques pour les troubles de comportement permettent de considérer comme perturbés des adolescents qui réagissent par un comportement antisocial à la pression de la part des camarades, à un environnement hostile ou aux mauvais traitements dans leur famille (Wakefield, Pottick, \& Kirk, 2002). Par exemple, une fille qui, pour tenter d'éviter l'intensification des agressions sexuelles de son beau-père, ment à ses parents sur le lieu de ses sorties, rentre tard malgré l'interdiction et puis, épuisée durant le jour, manque souvent les cours, ce qui entraîne la détérioration de son rendement scolaire, peut être étiquetée comme souffrant de troubles comportementaux (critères 11, 13 et 15). Les enfants contestataires ou ceux qui se retrouvent en mauvaise compagnie, manquent les cours et se livrent de façon répétitive au vol à l'étalage et au vandalisme sont également candidats au diagnostic. Toutefois, attestant de ces problèmes, un paragraphe dans l'article «Caractéristiques liées à la culture, à l'âge et au sexe » concernant les troubles de comportements mentionne qu' " en accord avec la définition du trouble mental selon le DSM, le diagnostic de " trouble du comportement » ne devrait être appliqué que lorsque le comportement en question est symptomatique d'une dysfonction sous-jacente chez l'individu et non simplement une réaction au contexte social immédiat »; on y note qu' " il peut être utile au clinicien de considérer le contexte social et économique dans lequel les comportements indésirables se sont produits »(DSM-TR, p. 96). Si ces idées avaient été incorporées aux critères diagnostiques, de nombreux diagnostics qui se sont révélés être des faux positifs auraient pu être évités. Malheureusement, dans les contextes épidémiologiques et de recherche, de telles nuances textuelles sont de toute évidence ignorées.

Le trouble d'anxiété de séparation est diagnostiqué chez les enfants sur la base de symptômes indiquant une anxiété excessive et inappropriée, relativement à la tranche d'âge, anxiété qui dure au moins quatre semaines par suite de la séparation d'avec ceux auxquels l'individu est attaché. Les symptômes (par exemple, la détresse excessive lorsque survient la séparation, la peur qu'un événement puisse mener à la séparation, qu'un malheur puisse arriver aux personnes auxquelles il est attaché, le refus de se rendre à l'école à cause de la peur de la séparation, la réticence à rester seul ou sans la figure d'attachement 
principale, sont tout à fait le genre d'expériences vécues par les enfants lorsqu'ils ont des réactions normales intenses face à la séparation. Les critères ne fournissent donc à l'utilisateur aucune procédure pour distinguer d'une part, un véritable trouble, dans lequel les réactions à la séparation sont déclenchées de façon inappropriée, et, d'autre part, les réactions normales aux menaces inhabituelles perçues par l'enfant quant à ses liens primordiaux à un parent peu fiable, ou à d'autres perturbations sérieuses. Par exemple, dans une étude auprès des enfants du personnel militaire de trois bases différentes (Bickman et al., 1995) qui s'est déroulée durant l'opération "Tempête du désert ", alors que de nombreux parents partaient pour le Moyen-Orient et que les enfants savaient que ceux-ci pouvaient être tués ou blessés, le degré d'angoisse de séparation était suffisamment élevé chez de nombreux enfants pour qu'ils remplissent les conditions requises pour éprouver un " trouble d'angoisse de séparation ", selon les critères du DSM (comparativement à des réactions à la séparation typiques de leur âge, leurs réactions étaient « excessives »). Mais, en fait, ils avaient des réactions proportionnées à un environnement très inhabituel dans lequel être inquiets que leurs parents ne puissent pas revenir était réaliste (A. M. Brannan, communication personnelle). Les enfants normaux dont les liens affectifs sont menacés dans la réalité peuvent ainsi être traités tout comme s'ils avaient des réactions d'attachement pathologiques, alors qu'on devrait aborder la question de leur besoin d'attachement réel.

Un diagnostic du DSM pour abus de substances, qui traite des conséquences négatives de l'usage de drogues, requiert l'un ou l'autre de ces trois critères : une piètre exécution de son rôle au travail ou à la maison occasionnée par l'usage de substances ; l'usage de substances dans des circonstances dangereuses, comme la conduite sous l'influence de l'alcool ; des problèmes judiciaires répétés liés à l'utilisation persistante de la substance malgré des problèmes sociaux ou interpersonnels récurrents dus à son usage, comme des querelles avec les membres de la famille sur les conséquences de l'intoxication. Ces critères ne sont pas seulement sans validité comme tels en tant qu'indicateurs du trouble, mais ils sont également en contradiction avec la définition même du trouble mental du DSM, qui affirme que les « symptômes " ne doivent pas être occasionnés par des conflits avec la société. Les arrestations pour activités illégales ainsi que l'usage de stupéfiants malgré la désapprobation de la famille sont exactement le genre de conflits sociaux qui sont insuffisants pour diagnostiquer un trouble, suivant la définition du DSM. Si vous continuez à consommer malgré des disputes de couple à propos de l'alcool ou de la drogue, cela est en soi suffisant, selon le DSM, pour diagnostiquer un abus de substance. Si vous buvez ou fumez de la marijuana, votre conjoint ou votre conjointe peut produire en vous un trouble mental simplement en se querellant avec vous à ce propos et vous guérir en fermant les yeux sur votre intoxication! Être arrêté plus d'une fois pour conduite en état d'ébriété est également suffisant pour le diagnostic, de telle sorte que le statut diagnostique d'un individu dépendra de la diligence de la police locale. Comme pour le critère 
"d'usage dangereux ", un très grand nombre de gens conduisent sous l'influence de l'alcool pour toutes sortes de raisons stupides et n'ont pas besoin d'un trouble mental pour le faire.

\section{Limitation du critère « cliniquement significatif » pour éliminer les faux positifs}

La tentative la plus notable du DSM de progresser généralement concernant la question des faux positifs est que, tout au long du manuel, l'exigence que les symptômes doivent occasionner " une importante détresse ou une détérioration dans le fonctionnement social, scolaire ou occupationnel » — que j'appelle " le critère "cliniquement significatif" » $\left(\mathrm{CCS}^{2}\right)$ — ou un équivalent approximatif, a été ajouté à environ la moitié des ensembles de critères. Le CCS vise à fixer un seuil de détérioration ou de détresse pour les diagnostics afin d'éliminer les faux positifs là où il y a préjudice minimal pour l'individu, et dans quelques cas il augmente effectivement la validité de ces diagnostics. Toutefois, exiger une détresse ou une détérioration " cliniquement significative » comme critère pour distinguer les troubles des conditions normales est un critère circulaire, parce que " cliniquement significatif » dans ce contexte peut uniquement signifier que la détérioration est suffisamment importante pour indiquer l'existence d'un trouble. L'expression n'offre aucune orientation claire afin de décider si le degré de détérioration est ou non suffisant pour indiquer un trouble.

De plus, elle ne tient pas compte d'un grand nombre de faux positifs potentiels, en particulier ceux qui peuvent engendrer une condition préjudiciable, mais pas de dysfonction (Spitzer et Wakefield, 1999), par exemple l'enfant normal dans un environnement menaçant, dont le comportement agressif satisfait au critère de trouble du comportement. La jeune fille normale menacée par le petit caïd de l'école et dont l'apparent " mutisme " rencontre le critère de mutisme sélectif parce qu'elle ne parle pas à l'école éprouve bel et bien de la détresse ; la détérioration significative de son fonctionnement et constitutive de ses réactions normales n'est donc pas exclue du diagnostic par le CCS. Bien qu'un problème épineux persiste ici quant au point à partir duquel les motivations deviennent si intenses et rigides qu'elles en sont pathologiques, il semble clair que dans certains cas (par exemple, ne pas parler pour éviter d'être battue par un voyou) elles peuvent être parfaitement normales, même si elles amoindrissent les résultats et sont embarrassantes.

L'aspect peut-être le plus problématique du CCS est qu'il reflète une mauvaise compréhension du problème central sous-tendant les faux positifs et, de ce fait, une mauvaise orientation de l'effort. Le CCS est basé sur la supposition que la façon de s'assurer qu'une condition est pathologique est de s'assurer qu'elle cause une détresse ou une détérioration significative du rôle, une supposition qui ne coïncide pas avec la pratique diagnostique plus générale

2. Note du traducteur : je traduis « clinical significance criterion » par « critère «cliniquement significatif» "; l'acronyme «CCS » remplace ici le « CSC » du texte original. 
en médecine. Les faux positifs du DSM sont le plus souvent attribuables non à l'incapacité des symptômes à atteindre un seuil de nuisance, mais à l'incapacité des critères symptomatiques à indiquer la dysfonction sous-jacente. Ainsi, élever le degré des malaises comme la détresse ou la détérioration n'est pas suffisant pour distinguer les troubles des états normaux. Il y a deux bons indicateurs de cette incapacité dans le DSM lui-même. Premièrement, le faux positif potentiel le plus évident dans le manuel, soit le deuil sans complication, distinct du trouble de dépression majeur, doit être pris en considération dans une clause d'exception spéciale additionnelle et n'est pas éliminé par le CCS qui est ajouté à l'ensemble des critères, parce que le chagrin habituel peut susciter autant de détresse et être aussi incommodant pour le rôle que la dépression pathologique. Deuxièmement, quoique le CCS soit ajouté aux critères des troubles de comportement, le DSM ajoute malgré tout une note textuelle disant que les enfants peuvent satisfaire au critère et néanmoins n'être pas perturbés, parce que leur comportement antisocial peut ne pas être attribuable à une dysfonction, mais plutôt être une réaction normale à un environnement problématique. En clair, le CCS n'aborde pas les problèmes reliés à la dysfonction.

Comme l'illustre, je l'espère, cette discussion, le pouvoir explicatif de l'analyse DP est selon toute vraisemblance supérieur aux explications rivales $\mathrm{du}$ " trouble ». L'analyse fournit aussi la base potentielle efficace d'une critique positive des pratiques diagnostiques en psychiatrie qui retient ce qui est sensé et rejette ce qui est excessif dans les applications du terme de «trouble » à certaines conditions mentales et comportementales. En nous fournissant une position ferme pour critiquer la psychiatrie, l'analyse nous aidera selon toute vraisemblance à clarifier les fondations conceptuelles de celle-ci, ce que n'ont pas fait les critiques foucaldiennes et szasziennes.

(Traduction Sébastien Cloutier)

\section{Bibliographie}

Ausubel, D.P. « Personality Disorder is Disease ", American Psychologist, 16, 1971, p. 59-74.

Boorse, C. "On the Distinction Between Disease and Illness ", Philosophy and Public Affairs, 5, 1975, p. 49-68.

- "What a Theory of Mental Health Should be ", Journal for the Theory of Social Behavior, 6, 1976a, p. 61-84.

—. "Wright on Functions », Philosophical Review, 85, 1976b, p. 70-86.

_. "Health as a Theoretical Concept ", Philosophy of Science, 44, 1977.

Cummins, R. "Functional Analysis », The Journal of Philosophy, 72, 1975, p. 741765.

Elster, J. Explaining Technical Change, Cambridge, Cambridge University Press, 1983.

Foucault, M. Madness and Civilization: a History of Insanity in the Age of Reason (trad. de R. Howard), New York, Pantheon, 1965.

- History of Sexuality. An Introduction, vol. 1, New York, Pantheon, 1978.

Gorenstein, E.E. « Debating Mental Illness : Implications for Science, Medicine, and Social Policy », American Psychologist, 39, 1984, p. 50-56. 
Hempel, C.G. « The Logic of Functional Analysis », dans C. G. Hempel, Aspects of Scientific Explanation and Other Essays in the Philosophy of Science, New York, the Free Press, 1965, p. 297-300.

Horwitz, A.V. « The Social Control of Mental Illness », Orlando, Fla., Academic Press, 1982.

Houts, A.C. "Harmful Dysfunction and the Search for Value Neutrality in the Definition of Mental Disorder : Response to Wakefield, Part 2 ", Behavior Research and Therapy, 39, 2001, p. 1099-1132.

Kendell, R.E. "The Concept of Disease and its Implications for Psychiatry ", British Journal of Psychiatry, 127, 1975, p. 305-315.

_. "What are Mental Disorders ? ", dans A.M. Freedman, R. Brotman, I. Silverman et D. Hutson, dir., Issues in Psychiatric Classification : Science, Practice and Social Policy, New York, Human Sciences Press, 1986, p. 23-45.

Kirmayer, J.L. et A. Young. "Culture and Context in the Evolutionary Concept of Mental Disorder », Journal of Abnormal Psychology, 108, 1999, p. 446-452.

Klein, D.F. « A Proposed Definition of Mental Illness », dans R.L. Spitzer et D.F. Klein, dir., Critical Issues in Psychiatric Diagnosis, New York, Raven Press, 1978, p. 41-71.

Macklin, R. « Mental Health and Mental Illness : Some Problems of Definition and Concept Formation ", dans A.L. Caplan, H.T. Engelhardt Jr., et J.J. McCartney, dir., Concepts of Health and Disease : Interdisciplinary Perspectives, Reading, Mass., Addison-Wesley, p. 391-418, 1981.

Moore, M.S. «Discussion of the Spitzer-Endicott and Klein Proposed Definitions of Mental Disorder (Illness) ", dans R.L. Spitzer et D.F. Klein, dir., Critical Issues in Psychiatric Diagnosis, New York, Raven Press, 1978, p. 85-104.

Nagel, E. Teleology Revisited and Other Essays in the Philosophy and History of Science, New York, Columbia University Press, 1979.

Ruse, M. The Philosophy of Biology, Londres, London University Press, 1973.

Sadler, J.Z. "Horsefeathers : a Commentary on 'Evolutionary Versus Prototype Analyses of the Concept of Disorder' ", Journal of Abnormal Psychology, 108(3), 1999, p. 433-437.

Sarbin, T. " On the Futility of the Proposition That Some People be Labeled 'Mentally Ill' ", Journal of Consulting Psychology, 31, 1967, p. 447-453.

Sarbin, T. "The Scientific Status of the Mental Illness Metaphor ", dans S.C. Pong et R.B. Edgerton, dir., Changing Perspectives in Mental Illness, New York, Holt, Rinehart \& Winston, 1969.

Sedgwick, P. Psycho Politics, New York, Harper \& Row, 1982.

Spitzer, R.L. et J. Endicott. " Medical and Mental Disorder : Proposed Definition and Criteria ", dans R.L. Spitzer et D.F. Klein, dir., Critical Issues in Psychiatric Diagnosis, New York, Raven Press, 1978, p. 15-39.

Spitzer, R.L. et J.C. Wakefield. « DSM-IV Diagnostic Criterion for Clinical Significance : Does it Help Solve the False Positives Problem ? ", American Journal of Psychiatry, 156, 1999, p. 1856-1864.

Wakefield, J.C. "The Concept of Mental Disorder : on the Boundary Between Biological Facts and Social Values », American Psychologist, 47, 1992a, p. 373388.

—. "Disorder as Harmful Dysfunction : a Conceptual Critique of DSM-III-R's Definition of Mental Disorder », Psychological Review, 99, 1992b, p. 232-247. 
- « Limits of Operationalization : a Critique of Spitzer and Endicott's 1978 Proposed Operational Criteria for Mental Disorder », Journal of Abnormal Psychology, 102, 1993, p. 160-172.

- « Dysfunction as a Value-Free Concept : Reply to Sadler and Agich », Philosophy, Psychiatry, and Psychology, 2, 1995, p. 233-246.

—. " DSM-IV : Are We Making Diagnostic Progress ? ", Contemporary Psychology, 41, 1996, p. 646-652.

- "Diagnosing DSM, Part 1 : DSM and the Concept of Mental Disorder ", Behavior Research and Therapy, 35, 1997a, p. 633-650.

—_. "Diagnosing DSM, Part 2 : Eysenck 1986 and the Essentialist Fallacy ", Behavior Research and Therapy, 35, 1997b, p. 651-666.

- " Meaning and Melancholia : Why the DSM Cannot (Entirely) Ignore the Patient's Intentional System ", dans J.W. Barron, dir., Making Diagnosis Meaningful : Enhancing Evaluation and Treatment of Psychological Disorders, Washington, D.C., American Psychological Association, 1998, p. 29-72.

_. " Evolutionary Versus Prototype Analyses of the Concept of Disorder ", Journal of Abnormal Psychology, 108, 1999a, p. 374-399.

- - " "Disorder as a Black Box Essentialist Concept ", Journal of Abnormal Psychology, 108, 1999b, p. 465-472.

-. "The Measurement of Mental Disorder ", dans A.V. Horwitz et T.L. Scheid, dir., A Handbook for the Study of Mental Health, New York, Cambridge University Press, 1999c, p. 29-57.

—. "Aristotle as Sociobiologist : the 'Function of a Human Being' Argument, Black Box Essentialism, and the Concept of Mental Disorder ", Philosophy, Psychiatry, and Psychology, 7, 2000a, p. 17-44.

_. "Spandrels, Vestigial Organs, and Such : Reply to Murphy and Woolfolk's 'The Harmful Dysfunction Analysis of Mental Disorder' ", Philosophy, Psychiatry, and Psychology, 7, 2000b, p. 253-270.

- « Values and the Validity of Diagnostic Criteria : Disvalued Versus Disordered Conditions of Childhood and Adolescence ", dans J.Z. Sadler, dir., Descriptions o Prescriptions: Values, Mental Disorders, and the DSMS, Baltimore, John Hopkins University Press, 2002a, p. 148-164.

- « Fixing a Foucault Sandwich : Cognitive Universals and Cultural Particulars in the Concept of Mental Disorder ", dans K.A. Cerulo, dir., Culture in Mind : Toward a Sociology of Culture and Cognition, New York, Routledge, 2002b, p. 245-266.

—. "Dysfunction as a Factual Component of Disorder : Reply to Houts, Part 2 ", Behavior Research and Therapy, 41, 2003, p. 969-990.

Wakefield, J.C., et M. First. « Clarifying the Distinction Between Disorder and NonDisorder : Confronting the Overdiagnosis ('False Positives') Problem In DSM$\mathrm{V} »$, dans K.A. Phillips, M.B. First et H.A. Pincus, dir., Advancing DSM : Dilemmas In Psychiatric Diagnosis, Washington, D.C., American Psychiatric Press, 2003.

Wakefield, J.C., Pottick, K.J. et S.A. Kirk. "Should the DSM-IV Diagnostic Criteria for Conduct Disorder Consider Social Context ? ", American Journal of Psychiatry, 159, 2002, p. 380-386.

Woodfield, A. Teleology, Cambridge, Cambridge University Press, 1976.

Wright, L. "Functions ", Philosophical Review, 82, 1973, p. 139-168.

—. Teleological Explanations, Berkeley, CA, University of California Press, 1976. 